\title{
Obituary
}

\section{In memoriam: Vladimir Morozov (1945-2021)}

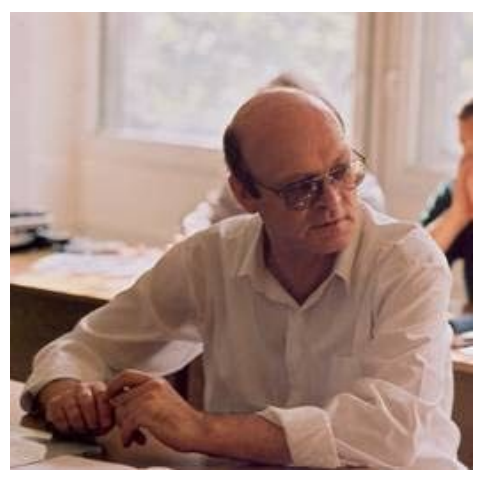

When this issue of the journal was being prepared for publication, we got a sad news: on November 30, 2021, our colleague, the longtime author and member of the Editorial Board of Condensed Matter Physics, Prof. Vladimir Georgievich Morozov passed away.

Vladimir Morozov was born on November 3, 1945, in Moscow to the family of a marine officer. In 1969 he graduated from the Physics department of M. Lomonosov Moscow State University. In 1973, one year after the completion of his postgraduate studies at Steklov Mathematical Institute of the Academy of Science of USSR, he defended his PhD thesis entitled "The questions of a quantum statistical theory of anisotropic ferro- and antiferromagnets at low temperature". In 1988 he received the degree of Doctor of Physical and Mathematical Sciences, having successfully defended his thesis "Statistical mechanics of nonlinear and non-equilibrium hydrodynamic fluctuations". From 1989 until the last days of his life he worked as a professor at the Department of Nanoelectronics in MIREA - Russian Technological University.

He is the author of over two hundred papers on the statistical theory of non-equilibrium processes, the dynamics of autowaves in active media, kinetic processes in semiconductors under the action of laser radiation, the non-equilibrium quantum field theory, kinetics of the relativistic plasma, the theory of open quantum systems, and many other papers in various branches of theoretical physics. His twovolume textbook "Statistical Mechanics of Non-equilibrium Processes", written in co-authorship with Profs. D. N. Zubarev and G. Röpke, is one of the best in this field of science, and indeed remains to be a hand-book for numerous theoretical physicists.

Since the mid-1980s, V. G. Morozov together with Prof. D. N. Zubarev, a colleague and a good friend of many of us, greatly contributed to launching a new promising area of research - the statistical physics of non-equilibrium processes, which later became one of the basic scientific activities at the Institute for Condensed Matter Physics. V. G. Morozov repeatedly visited our Institute, was a member of the Organizing Committee of our traditional conference "Statistical Physics: Modern Trends and Applications". Every meeting with him was very useful: he proved himself to be a very good lecturer and was always open to discussions.

Vladimir Morozov was a brilliant and profound scientist; he will remain in our memory as a person of encyclopedic knowledge, devoted to true science, a pattern of diligence and perfection of presentation of scientific results. We can surely say that he belongs to the constellation of outstanding theoretical physicists, descendants of the scientific school of M. M. Bogolyubov, who left a bright mark in science and expanded the frontiers of our knowledge.

The Editorial Board of Condensed Matter Physics 